\title{
Microscopical studies of necrotising scleritis. I. Cellular aspects
}

\author{
ROBERT D. YOUNG' AND PETER G. WATSON ${ }^{2}$ \\ From the 'Strangeways Research Laboratory, Cambridge; and the 'Department of Ophthalmology, \\ Addenbrooke's Hospital, Cambridge
}

SUMMARY Light and electron microscopy were used to examine tissue excised during surgery from eight patients with advanced destructive scleral disease. These comprised two cases of scleromalacia perforans, three cases of anterior necrotising scleritis alone or in conjunction with other systemic diseases, and three cases in which scleritis developed following ocular surgery. It was not possible to distinguish between these three categories by histological or cytopathological criteria. All showed extensive granulomatous infiltration of the conjunctiva, episclera, and sclera by plasma cells and lymphocytes. Mast cells were abundant throughout these inflamed tissues. Examination of scleral stroma from sites in advance of the granuloma revealed active fibroblastic cells in the absence of other inflammatory cells. Fibroblastic transformation of scleral cells may be one of the earliest events in scleral degradation during necrotising disease.

The sclera is a diffusely cellularised, poorly vascularised connective tissue which, however, may be subject to a variety of inflammatory disorders that dramatically alter its vascular pattern and cellularity. ${ }^{12}$

Studies of the vascular changes which occur in scleral disease ${ }^{3}$ show that in those forms of scleritis which do not lead to destructive changes there is a rapid vasodilatation or rapid flow throughout the inflamed area. However, if the inflammation is prolonged or very severe this rapid flow pattern gives way first to sluggish flow, then venular occlusion, and finally obliteration of the vascular network (Fig. 1). If the condition remains untreated, and sometimes even in spite of vigorous treatment, a gradual or rapid ulceration of the connective tissue matrix then occurs which, if allowed to progress, may involve other ocular structures (Fig. 2) and lead to loss of vision.

The sequence of events that lead to this severely painful necrotising process is still little understood. Although scleral disease does occur as an isolated condition, it is often a component of many multisystem connective tissue disorders which have an immunological basis. A local injury to the eye in the form of a surgical operation of any sort has frequently been found to induce scleral inflammation, particularly if the patient has high pathological circulating

Correspondence to Di R. D. Young, Strangeways Rescarch Laboratory, Wort's Causcway, Cambridgc CB1 4RN. immune complexes or has had a recurring infection. ${ }^{4}$ It can also follow herpes zoster infection at the site of the initial inflammation. Some patients with longstanding advanced seropositive rheumatoid arthritis develop a condition termed scleromalacia perforans, in which areas of sclera sequestrate without associated pain or inflammation.

In previous histopathological studies of necrotising scleritis $^{56}$ a chronic granulomatous infiltration of the

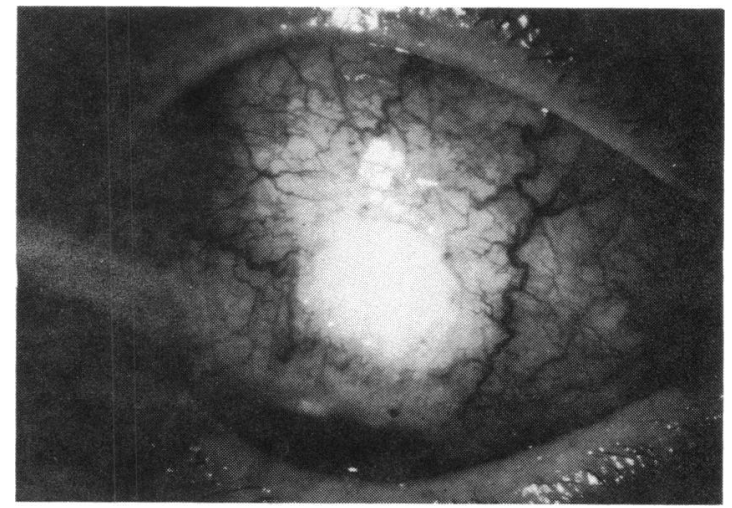

Fig. 1 Severe necrotising scleritis in a 57-year-old male Caucasian in which one area of episclera, and in this instance conjunctiva, has become avascular and is beginning to necrose. 


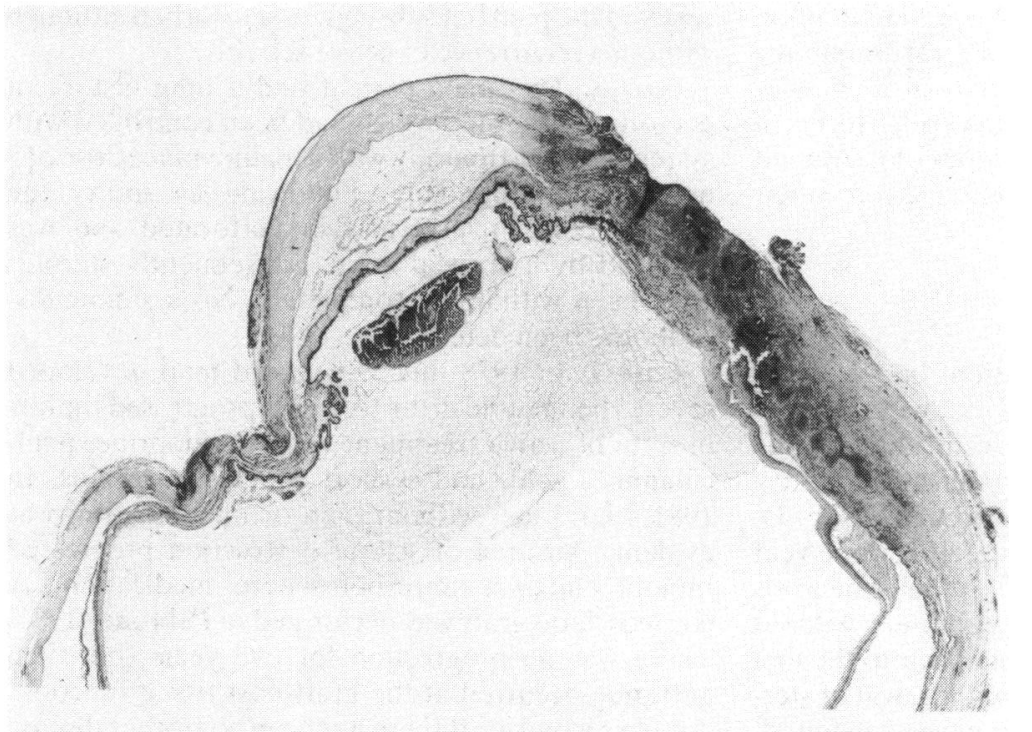

Fig. 2 Anterior necrotising scleritis uninfluenced by steroid treatment, which could not be given because of severe systemic bacterial infection. The scleral granuloma involves not only the whole of the anterior sclera but extends back into the ciliary body and choroid causing an intense uveitis.

scleral stroma was observed with destruction of the collagenous matrix (Fig. 3). The question arises whether tissue destruction is a direct result of granulomatous changes within the sclera or, alternatively, whether granuloma formation is secondary to and obscures some more subtle resorptive process. Previous studies have been unable to resolve between these two possibilities, largely because tissue for histopathological study normally becomes available only when the disease is in a late stage and tissue destruction is so advanced that reconstructive surgery or enucleation of the eye becomes necessary. Theoretically, because of the progressive nature of scleral ulceration, this problem can be overcome by careful selection and orientation of tissue excised from sites in advance of the ulcerating front. Events corresponding to early stages in the destructive process may then be examined.

The purpose of this study was to compare the clinical presentation with light and transmission electron microscopical findings in tissue obtained from eight patients who required surgery in the treat-

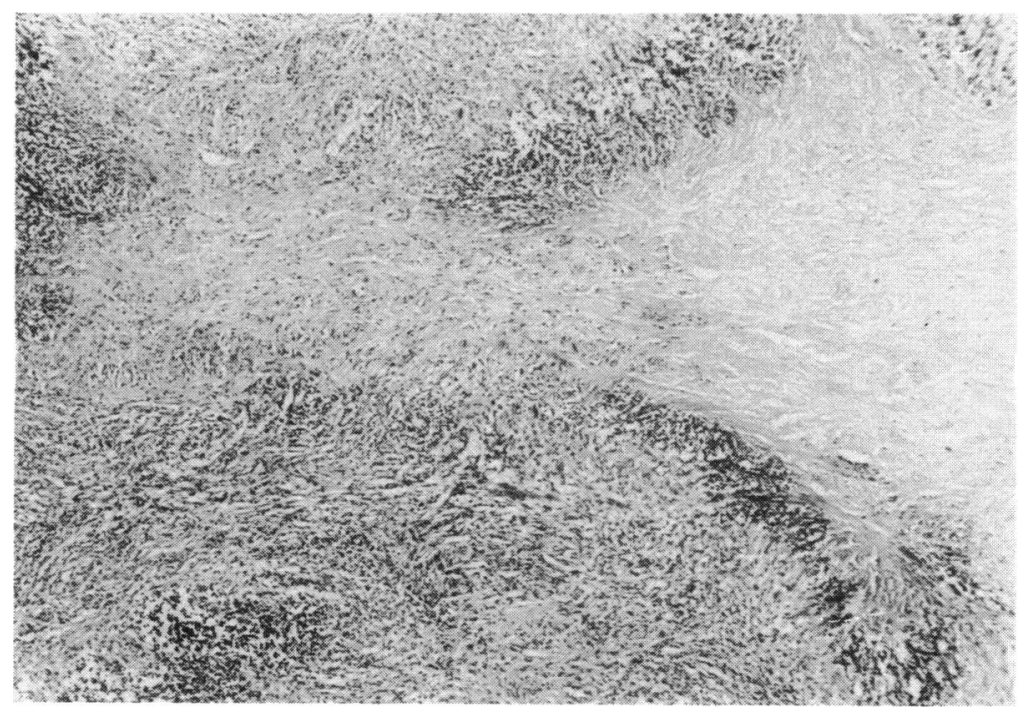

Fig. 3 Chronic granuloma in destructive scleral disease in which there is progressive infiltration of the scleral stroma by inflammatory cells. Normal scleral lamellae (at right) are undergoing degradation in advance of the granuloma (left). $(\times 35)$. 
ment of destructive scleral disease. Special attention was given to sites in advance of the scleral granuloma in order to identify any early cytopathological changes which could distinguish between the various forms of the disease and thus influence the types of treatment given or provide a clear indication as to their prognosis.

\section{Case histories}

Case 1. This 57-year-old male patient had a 2-year history of increasingly painful red eye with areas of vascular closure found by fluorescein angiography from first examination. In spite of intensive oral steroid therapy a large area of necrosis occurred (Fig. 1). After grafting this stabilised completely for one year without therapy, when a further episode of necrosis took place. This subsequently stabilised without therapy. Except for hyperglycaemia prior to the first operation, which disappeared on withdrawal of steroids, no other associated conditions have been found.

Case 2. This 65-year-old man had a 10-year history of red, painful eye following a serious bout of sinusitis and polyarthralgia. He had undergone two previous 'serious' operations about which no details were available. He had gout for many years treated with long term allopurinol. No evidence of Wegener's granulomatosis or other connective tissue disease was found. He presented with sclerouveitis and a very hazy vitreous which cleared after a short course of intensive steroid therapy. After three years he again presented with a red painful eye, very poor vision, and one area of necrotising scleritis. A scleral graft relieved the pain but subsequently resorbed although without a recurrence of active scleritis.

Case 3. This male patient had a long history of necrotising scleritis, which had been controlled with systemic steroid therapy with a maintenance dose of 5 $\mathrm{mg}$ prednisolone daily. Following an injury the thinned corneoscleral margin perforated and was replaced by a corneal inlay. Subsequently steroids have been withdrawn completely. No systemic disorder has been detected.

Case 4. In 1978 this 59-year-old man developed severe rheumatoid arthritis which progressed rapidly in spite of active treatment with azathioprine, penicillamine, gold, and a short course of steroids. In 1981 scleral loss without pain or inflammation was evident. An area of scleral destruction progressed rapidly and was unresponsive to medication. A corneoscleral graft was performed in February 1981. There was no progression for two years, but then guttering occurred at the graft-host tissue junction. After a second graft there has been no further disease activity.

Case 5. This male patient had a four-year history of active, progressive rheumatoid arthritis and rapidly became intolerant to all forms of therapy. Scleral destruction commenced without other symptoms. The defect was repaired with corneal tissue. There has been no further progression of this lesion, but areas of the sclera in this and the fellow eye have now become affected.

Case 6. This 56-year-old woman had undergone two squint operations as a child and her eyes had become increasingly divergent with age. A further
Fig. 4 Light micrograph of conjunctiva and sclera adjacent to scleral defect in case 4. Conjunctival epithelium is of variable thickness with a downgrowth to the level of the sclera at a site of previous conjunctival perforation (arrow). The conjunctival stroma is densely infiltrated with plasma cells and lymphocytes. Tenon's capsule and the episclera are obscure. The sclera contains a deep cellular infiltrate with loss of structure while the outer lamellae are intact. $(\times 98)$.

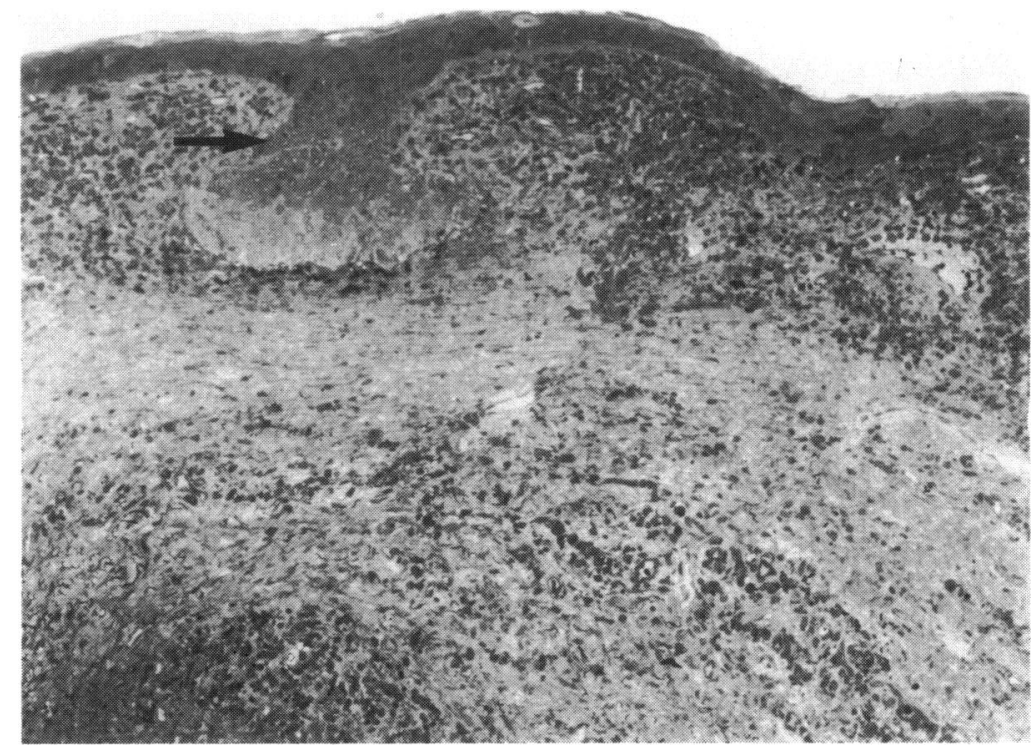




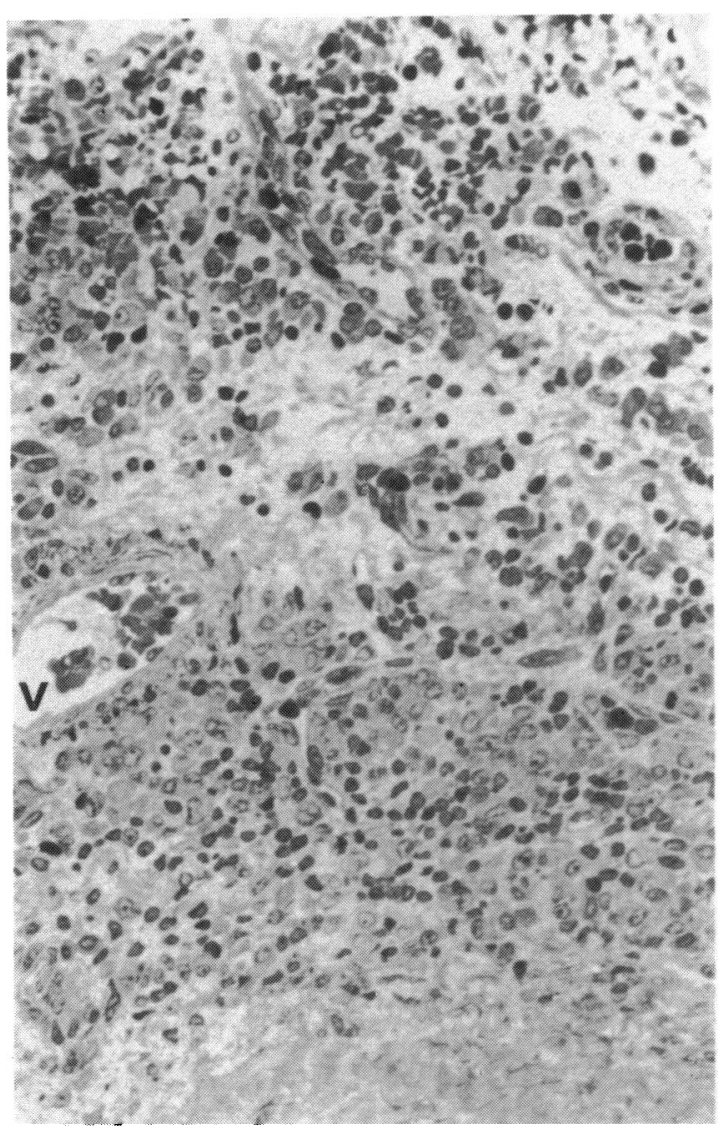

Fig. 5 The conjunctival stromal infiltrate in case 5 extends into the episclera. Plasma cells and lymphocytes are densely packed around an episcleral vessel $(\mathrm{v}) .(\times 345)$.

operation was performed on the muscles giving a reasonable corrective result. However, six weeks later a severe, progressive scleritis developed at the site of surgery on the lateral rectus. The pain and inflammation responded poorly to medication, so the affected area was replaced by a corneal graft. The pain disappeared, and there has been no recurrence. No stitches were found in the lesion. She has no other systemic disorder.

Case 7. In 1969 at the age of 32 this male patient had heterochronic cyclitis. He did not develop significant complications until 10 years later when, over a period of a few months, cataract and glaucoma occurred. Pars plana lensectomy caused an acute rise in intraocular pressure. This responded to medication, but the eye remained red and painful with gaping of the corneoscleral wound. Scleral inflammation extended away from this site during the following two months in spite of intensive systemic steroid and immunosuppressive therapy. The inflamed area was

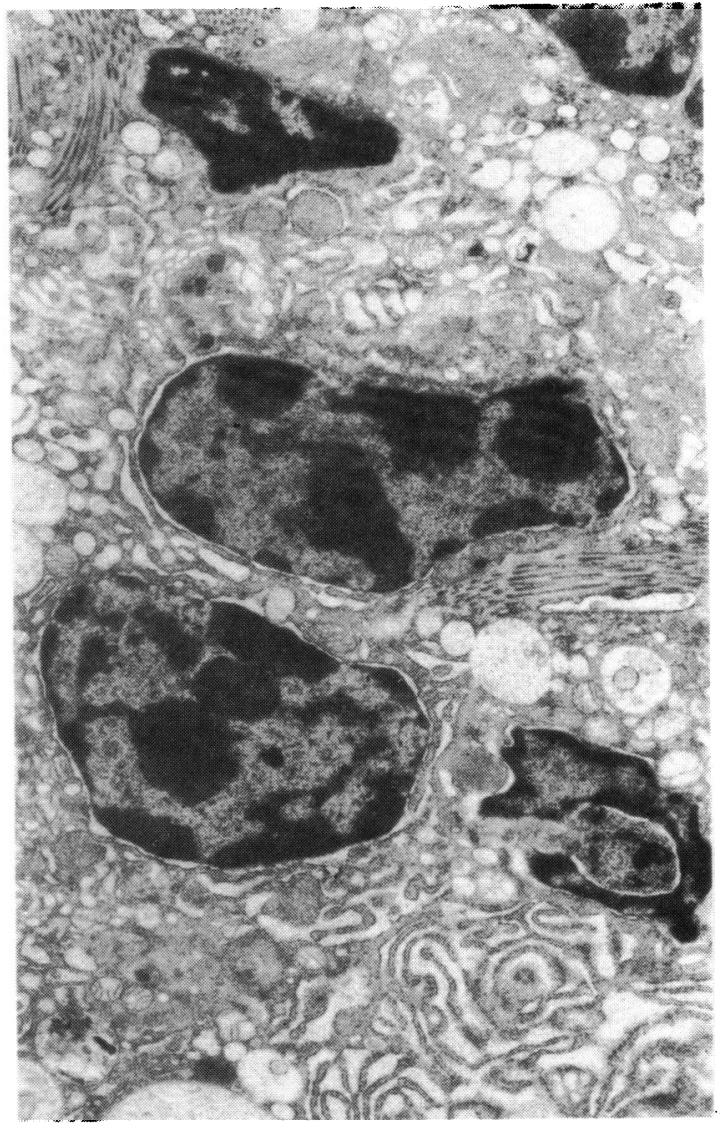

Fig. 6 Electron micrograph of deep episcleral/scleral stroma from case 7 showing advanced degeneration of plasma cells by apoptosis. $(\times 7500)$.

replaced with a scleral graft, and there has since been no recurrence or need for systemic therapy. Pupil block glaucoma has been treated by two laser iridectomies.

Case 8. This female patient developed retinal detachment with symptoms suggesting a rhegmatogenous detachment in which no hole could be found. She was treated with encircling cryotherapy and a circumferential band. The operation was followed by an episode of severe 'band pain' which resolved over a period of six weeks. However, the retinal detachment did not flatten. Further examination again failed to reveal a hole, but the sclera was found to be thickened and congested. On removing the band scleral inflammation was found to have arisen under the band and extended about $3 \mathrm{~mm}$ either side of it. In one area the sclera was almost absent and this area was patched. She was given systemic steroids following this procedure, and the detachment eventually resolved without further pain. 


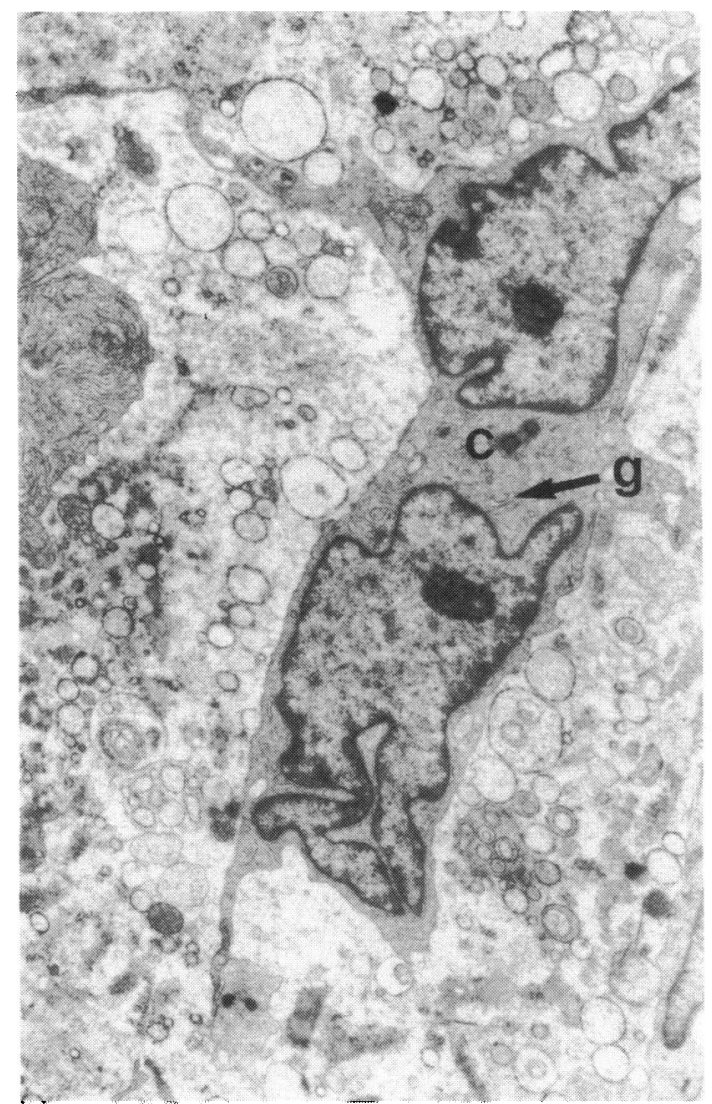

Fig. 7 Electron micrograph showing an active fibroblast containing centrioles $(\mathrm{c})$ golgi $(\mathrm{g})$ and irregular nucleus surrounded by RER saccules, the product of plasma cell degeneration in the episclera of case $4 .(\times 6000)$.

\section{Materials and methods}

Tissue excised during surgery was transferred immediately to $2.5 \%$ glutaraldehyde in $0.2 \mathrm{M}$ sodium cacodylate buffer containing $3 \mathrm{mM}$ calcium chloride at $\mathrm{pH} 7.2$ and fixed for two to three hours. After rinsing in buffer at $4^{\circ} \mathrm{C}$ it was immersed in buffered $1 \%$ osmium tetroxide for one hour, stained en bloc in $0.5 \%$ uranyl acetate, dehydrated in ethanol, cleared in propylene oxide, and embedded in Araldite.

Sections 1 to $2 \mu \mathrm{m}$ thick were cut on an LKB ultramicrotome and stained in $1 \%$ aqueous toluidine blue for light microscopy. Ultrathin sections were stained in alcoholic uranyl acetate and lead citrate and examined in a Philips 201 C electron microscope.

OBSERVATIONS

The cytopathology of necrotising scleritis in tissue from these eight patients reflects a varied degree of inflammation and cellular infiltration both spatially

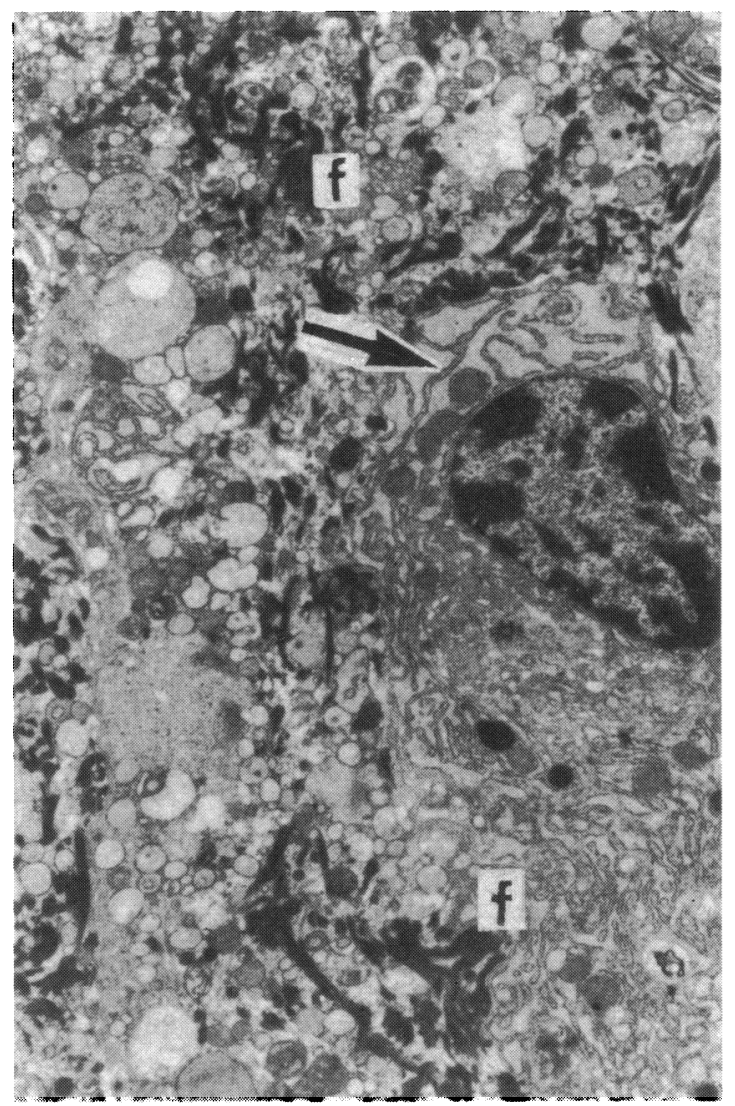

Fig. 8 Electron micrograph of episclera in case 6 shows fibrin deposition (f) and a mature plasma cell with dilated RER (arrow) in the stroma close to a small capillary (not shown $).(\times 6650)$.

with respect to the proximity of the scleral defect and quantitatively in relation to the extent and severity of scleral destruction. Qualitatively, however, the nature of the inflammatory infiltrate associated with these three clinically, and perhaps aetiologically, distinct forms of scleral disease was very similar. Necrotising scleral disease is therefore described according to its effects upon the structure of the successive connective tissue coats of the eye.

Conjunctiva. In all eight patients the conjunctiva displayed an intense inflammatory reaction with a dense stromal cell infiltrate, which in four patients resulted in an almost two-fold increase in stromal thickness. Adjacent to the scleral lesion the structure and organisation of the epithelium appeared normal, but overlying the lesion diverse epithelial changes were recorded. These varied from areas of partial or full thickness epithelial loss to those areas in which there were squamous changes and hypertrophy, where lymphocytes and neutrophils had migrated 

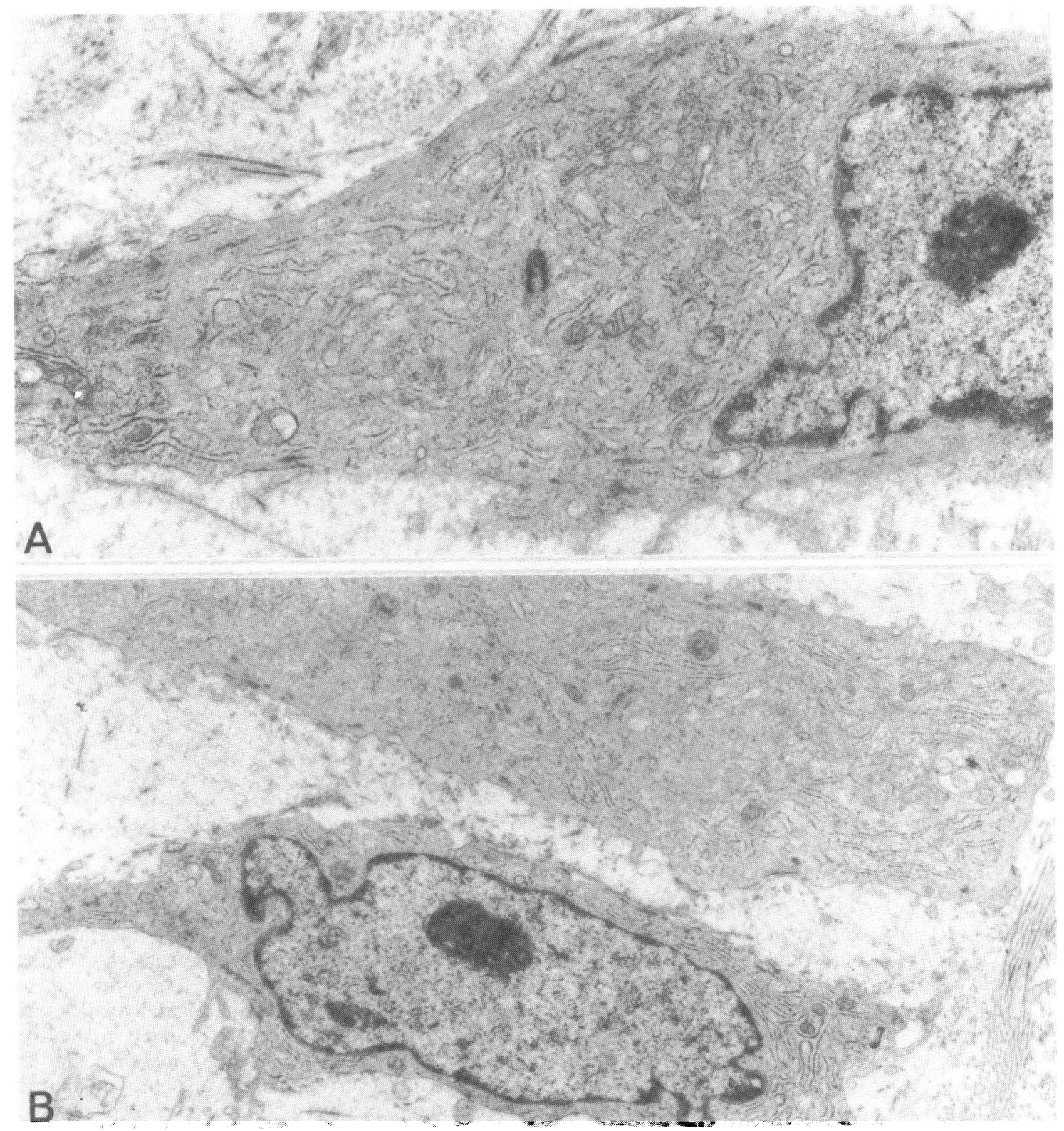

Fig. 9 Electron micrograph of scleral stroma from case 7 adjacent to scleral defect showing active fibroblasts in absence of invasive inflammatory cells. $(\mathrm{A}, \times 13500, \mathrm{~B}, \times 9000)$.

outwards through the basal lamina. The conjunctival contour was highly irregular overlying the scleral defect. Major erosions of the deep sclera in scleromalacia perforans were totally denuded of conjunctival tissue which was replaced by a fine film of fibrin and cell debris. Small areas of conjunctival perforation were filled with deep plugs of epithelium and intact basal lamina which formed downgrowths to the level of the episclera (Fig. 4).
Close to the site of scleral degradation the diffuse fine collagen fibrils of the conjunctival stroma were totally obscured by massed aggregations of plasma cells and lymphocytes. Cells were packed tightly into clusters sometimes reminiscent of the organisation in lymphoid follicles. At sites adjacent to the scleral granuloma early stages of stromal infiltration in the conjunctiva were seen as perivascular accumulations of lymphocytes and plasma cells usually with one or 


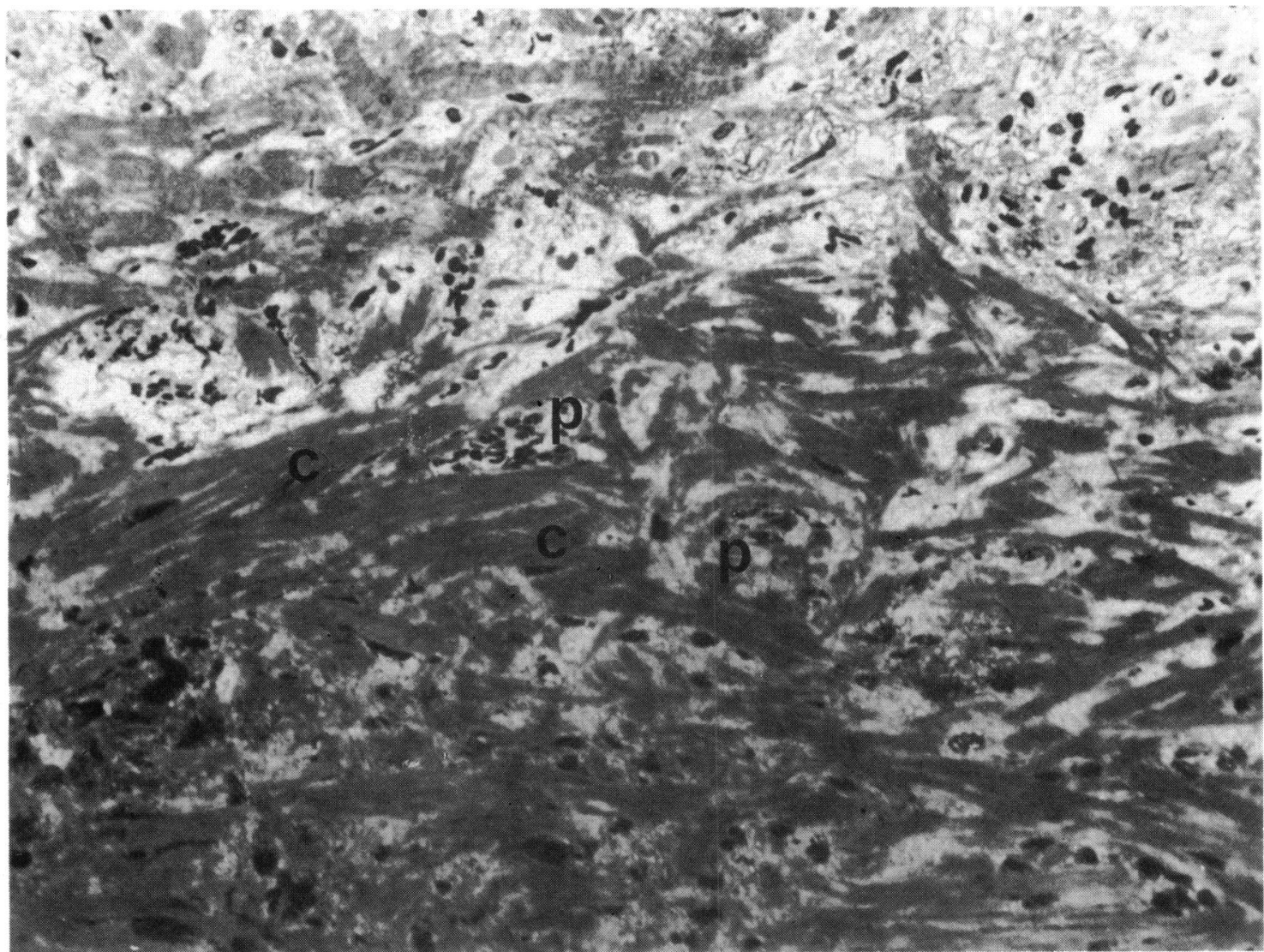

Fig. 10 Light micrograph of sclera in case 2 at the junction between granuloma (top) and scleral lamellae in which groups of pyknotic cells $(\mathrm{p})$ are contained in pockets of apparently intact collagen fibres $(\mathrm{c}) .(\times 280)$.

more attendant mast cells. Only at advanced stages of infiltration closely overlying scleral destruction were macrophages and neutrophils abundant in the conjunctival stroma.

Tenon's capsule and episclera. Tenon's capsule, the fine dense collagenous substratum deep to the conjunctiva, becomes obscure in the eyes of elderly people. In tissue from patients with necrotising scleritis Tenon's capsule was indistinguishable from the episclera. The episclera, a collagen fascia containing few cells, is distinct from the scleral stroma in normal eyes by virtue of its densely packed, fine fibrils and is easily located by the position of the superficial and deep episcleral plexuses of capillaries which invest the tissue and ramify within it. In all eight cases studied the conjunctival inflammatory infiltrate extended throughout the episclera displacing the vascular networks. Particularly dense accumulation of lymphocytes and plasma cells was located around episcleral capillaries close to the scleral defect (Fig. 5). Here cellular infiltration was sufficient to obliterate the episcleral stroma. In five cases scleral necrosis extended outward to involve the episclera. Apoptotic degeneration of plasma cells was seen at these sites involving rupture of cell membranes and release of organelles including Russell bodies and numerous saccules of ribosome-studded membrane (RER) (Fig. 6). Mast cells and lymphocytes often appeared intact surrounded by cell debris and matrix degradation products. Fibroblastic cells were numerous in the episcleral region overlying scleral lesions (Fig. 7). These cells contained many lysosomes, RER, and elaborate Golgi bodies. Fibrin deposition was a prominent feature of perivascular episcleral tissue (Fig. 8) with numerous tufted fibrils scattered among plasma cell debris and orientated along the collagen fibril bundles.

Scleral stroma. The dense collagenous scleral stroma also contains few cells in normal eyes. The stromal fibrocytes are attenuated in shape and lie along the lamellar interfaces. They appear metabolically inactive and, apart from a conspicuous 

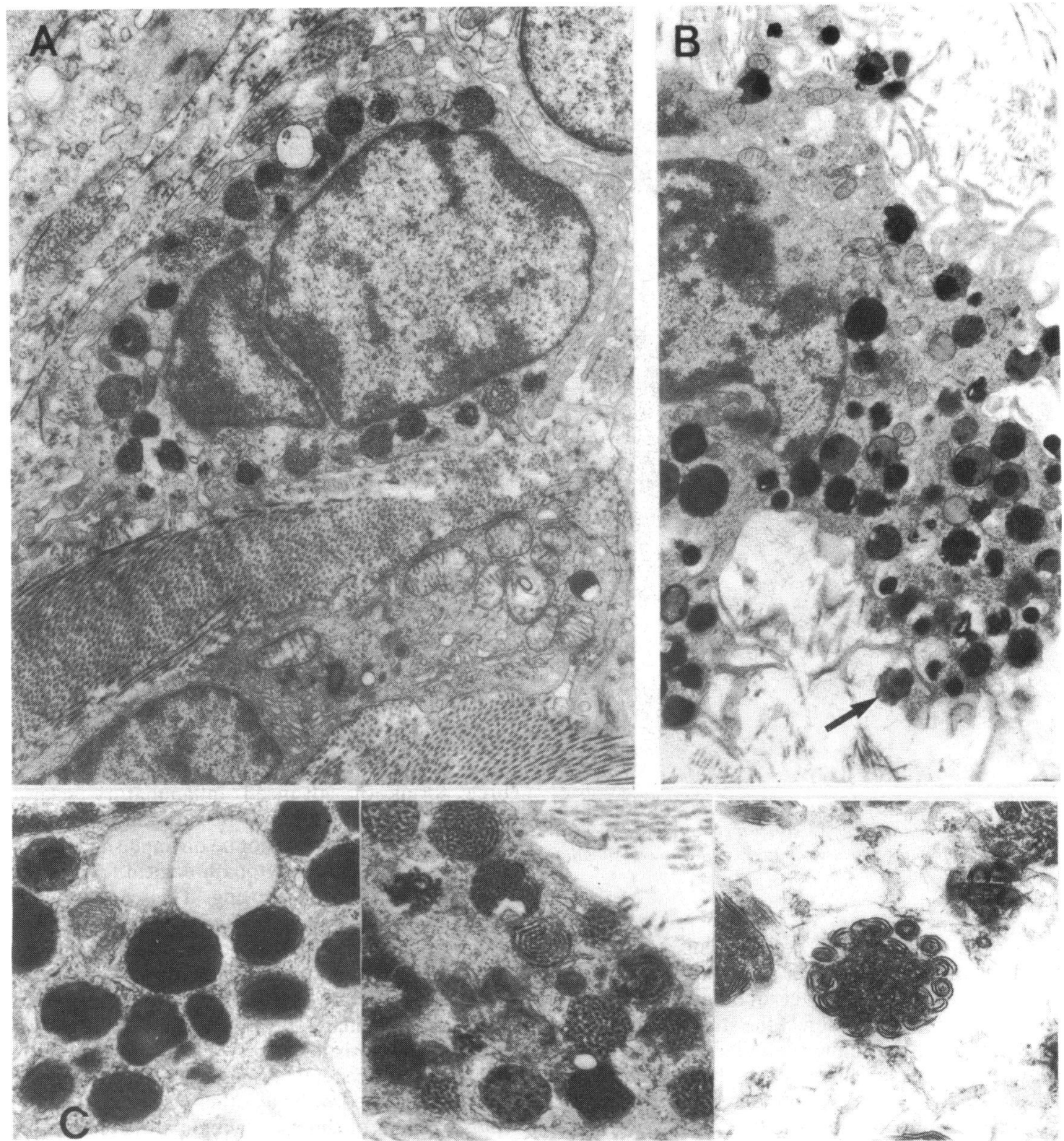

Fig. 11 Electron micrographs showing mast cells in scleral stroma. A. Mast cell in association with active stromal fibroblast shows swelling of cytoplasmic granules. $(\times 11250)$. B. Apparent extracellular granule release $($ arrow $)$ from a mast cell in a zone of stromal destruction. $(\times 10800)$. C. Variations of granule ultrastructre in scleral mast cells. (From left to right: $\times 20250$, $\times 19800$ and $\times 42000)$.

elongate nucleus, contain few organelles and relatively sparse cytoplasm. In contrast stromal cells in tissue from these patients were fibroblastic exhibiting increased size with branching peripheral pseudopodia. In addition they contained more diverse organelle systems with numerous mitochondria, prominent Golgi, and RER (Fig. 9). Centrioles with projecting cilia and increased numbers of lysosomal granules were also characteristic of these cells. Fibroblastic changes were located in scleral cells often distal to the advancing lesion within tissue which appeared normal by light microscopy. Electron microscopy revealed, however, that the scleral matrix surrounding active stromal cells, distant to the 


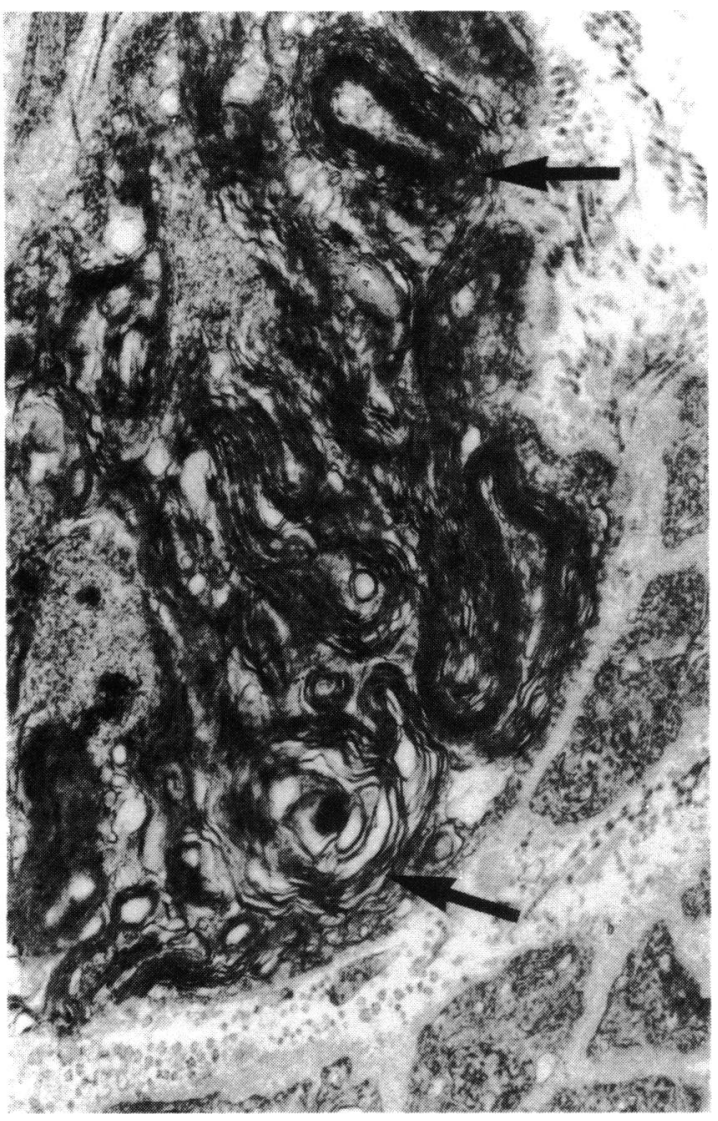

Fig. 12 A myelinated nerve axon traversing a scleral lesion in case 4 displays degenerative changes in the myelin sheath (arrows). (x16500).

granuloma, was already undergoing resorption. ${ }^{7}$ These changes appeared below conjunctival tissue showing widely ranging levels of inflammation.

Nearer to the scleral defect the stroma was swollen by granulomatous changes similar to those in the episclera with displacement of the lamellae by dense clusters of lymphocytes and plasma cells. Macrophages and multinucleate giant cells were also present. Cell and matrix necrosis was evident where the inflammatory infiltrate was most intense. Pockets of necrotic cells consisting of pyknotic nuclei and degenerating fibroblasts and plasma cells were enclosed by seemingly intact collagen bundles in three cases (Fig. 10). Mast cells were usually identified at these sites and, in addition, in the presence of phagocytic neutrophils and macrophages where total liquefaction of the stromal matrix with loss of collagen fibrils was evident.

Mast cells were generally rounded or fusiform in shape with central nuclei, some with nucleoli, and prominent peripheral heterochromatin. They were readily identified by their numerous veil-like projections around the circumference of the cell membrane and by the electron-dense granules scattered throughout the cytoplasm (Fig. 11). These showed variable fine structure. Most were round or spherical in shape and membrane bound. Some contained fine whorls or scrolls. In some cells granule size ranged from $0 \cdot 3$ to $0.7 \mu \mathrm{m}$ diameter those of larger size containing more diffusely stained contents. Only rarely was rupture of mast cell membranes and extracellular release of granules seen (Fig. 11).

In one case myelinated nerve fibres, presumably branches of the long posterior ciliary nerves, were located traversing the scleral stroma close to the zone of inflammation. Degenerative changes within the fibres ranged from disorganisation, vacuolation and rupture of the axonal myelin sheaths, to death of Schwann cells, and destruction of axons (Fig. 12).

In scleromalacia perforans a process of large scale removal of sclera was illustrated by sloughing of dense plaques of necrotic tissue (Fig. 13). This dramatic phenomenon appeared in association with full thickness loss of conjunctiva and the failure of conjunctival epithelium to resurface the exposed sclera. A dense scleral infiltrate was found below the sequestrating tissue.

\section{Discussion}

Three forms of advanced inflammatory scleral disease, which appeared to be clinically or aetiologically separable, could not be distinguished in this study by histological or cytopathological features of tissue removed during grafting. This concurs with earlier histological studies of enucleated eyes with presumed clinically disparate forms of scleral inflammation also in the late stages of the disease. ${ }^{68}$ Necrotising scleritis which follows intraocular surgery appears to be similarly indistinguishable in its pathological characteristics from necrotising scleritis associated with systemic diseases and that occurring alone unassociated with any other disease.

Observations of chronic granulomatous changes in the scleras of these eight cases are in general agreement with earlier reports. ${ }^{56}$ Infiltration of inflammatory cells into the scleral stroma may occur from three sources: the conjunctival and episcleral capillaries, the choroidal circulation across the lamina fusca, and the transscleral vessels themselves.

The cellular infiltrate consisted largely of plasma cells and lymphocytes indicating a localised immune response. Macrophages and giant cells were also present, although these cells were not as abundant as suggested in previous microscopical studies. In addition it was notable that neutrophils and eosino- 


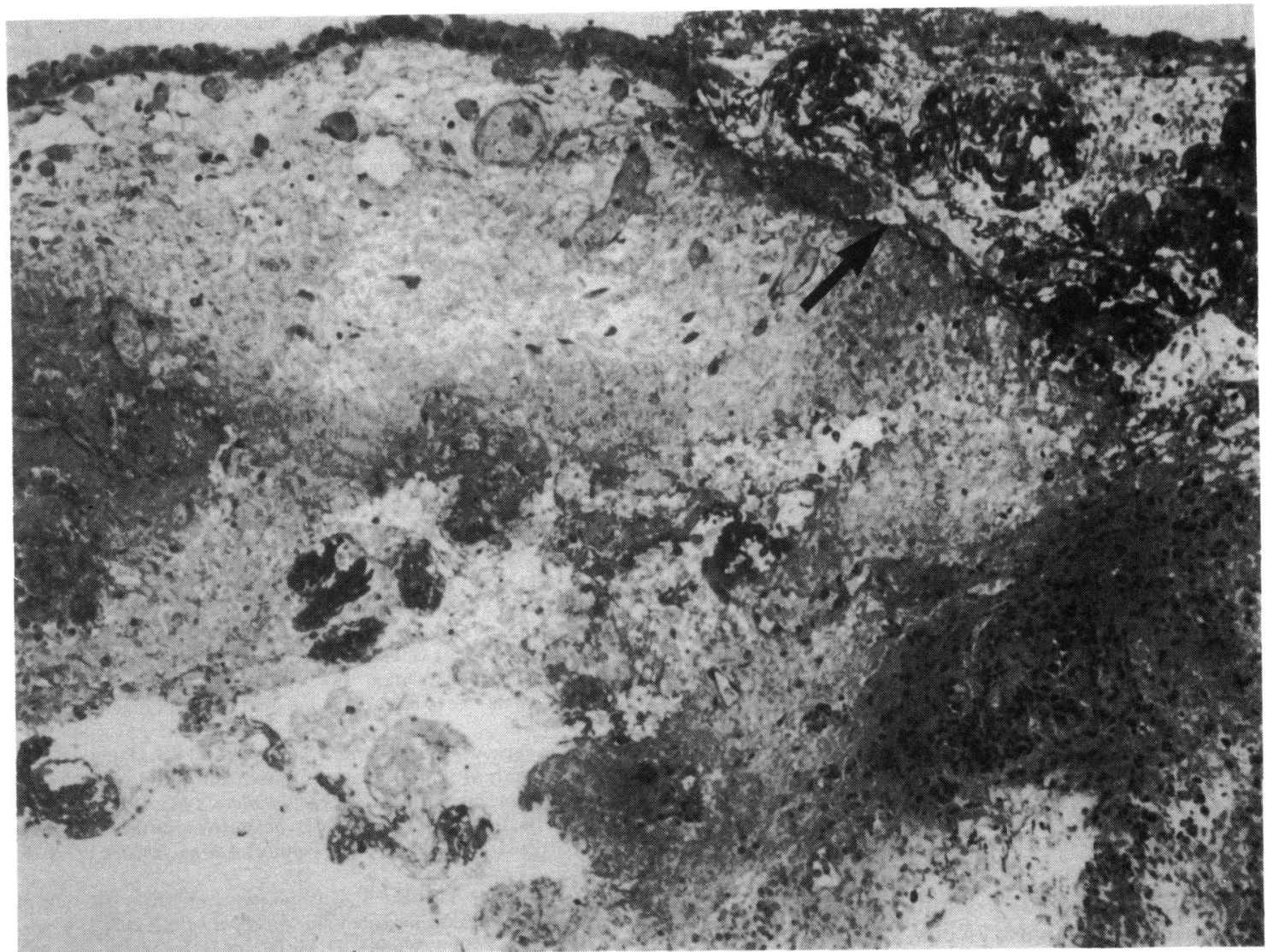

Fig. 13 Light micrograph from case 2, scleromalacia perforans illustrating loss of conjunctival tissue, incomplete resurfacing of sclera by conjunctival epithelium, and sloughing of a plaque of sequestered scleral tissue $($ arrow $) .(\times 140)$.

phils were present only in relatively small numbers throughout scleral tissue from these patients. This may be one result of the intensive systemic steroid therapy to which all eight patients had been subjected. ${ }^{9}$

The abundance of mast cells throughout the sclera at sites of inflammation may be an important observation which has not been previously reported. Mast cells are thought to function primarily in mediating inflammation through the release of vasoactive amines. Increased perivascular accumulation of mast cells in necrotising scleritis suggests that they may act as local regulators of inflammation by controlling vascular permeability and secretion of chemotactic factors. Mast cells are known also to be actively phagocytic, and while no clear evidence of phagocytosis was obtained here they may play a direct part in scleral resorption by this mechanism.

Variations in the ultrastructure of mast cell granules were seen which are representative of granule swelling. These changes are known to precede spontaneous degranulation of mast cells in vitro. "Similar changes have also been recorded in stimulated and unstimulated mast cells in rat ocular tissues." Extracellular release of mast cell granules was rarely observed and then only at sites of advanced tissue necrosis. Furthermore scleral mast cells showed no signs of the characteristic plasma membrane changes which appear to be a feature of anaphylactic or immediate hypersensitivity reactions."

In an attempt to determine whether scleral infiltration and granuloma formation precedes or follows resorption of the stromal matrix, or whether these events proceed simultaneously, we have examined tissue from sites in the path of the advancing granuloma. Here we have demonstrated activation of the stromal fibrocytes in conjunction with degradative changes in the surrounding collagenous matrix ${ }^{7}$ in the absence of infiltrating inflammatory cells. These may represent the earliest morphological events in scleral destruction suggesting that matrix resorption may indeed precede granuloma formation in the tissue. 
The stimulus for fibrocyte activation within the sclera is unknown. However, there is increasing evidence from cell and organ culture studies that diffusible factors released by a variety of tissues and cell types including intact synovium, lymphocytes, and macrophages, are able to stimulate connective tissue cells to degrade their matrices in vitro. ${ }^{12} 13$ Fibrocytes in uninflamed regions of the scleral stroma may, therefore, be stimulated to resorb their matrix by factors diffusing through the connective tissue milieu from either the adjacent granuloma or from inflammatory cells within the overlying conjunctival or episcleral tissue. In recent work using chondrocytes in culture it has been shown that mast cells may also be capable of the regulation of connective tissue cell metabolism via histamine $\mathbf{H}_{2}$ receptors borne on chondrocyte cell membranes. ${ }^{14}$ The influence of the products of inflammatory cells on the activity of scleral fibrocytes has yet to be investigated.

With the initiation of the resorptive process the scleral stroma and its component vascular and neural elements are gradually eroded. Structural damage to the sensory nerves traversing the sclera in conjunction with swelling and inflammation of the periaxonal stroma undoubtedly contribute to the severe pain encountered in this disease. The essential determinants of scleral destruction, therefore, appear to be, activation of scleral fibrocytes and resorption of pericellular matrix, infiltration of the scleral stroma by inflammatory cells, and prolonged local vaso-occlusion.
The authors gratefully acknowledge the support by a grant from the Wellcome Trust. Fig. 3 is reproduced with the permission of Harper and Row Publishers Inc., Maryland, USA.

\section{References}

1 Watson PG, Hazleman BL. The sclera and systemic disorders. London: Saunders, 1976.

2 Watson PG. The nature and treatment of scleral inflammation. Trans Ophthalmol Soc UK 1982; 102: 257-81.

3 Watson PG, Bovey E. Anterior segment fluorescein angiography in the diagnosis of scleral inflammation. Clin Ophthalmol in press.

4 Lyne A, Lloyd-Jones D. Necrotising scleritis after ocular surgery. Trans Ophthalmol Soc UK 1979; 99: 146-9.

5 François J. Ocular manifestations in collagenoses. Adv Ophthalmol 1970; 23: 1-54.

6 Sevel D. Necrogranulomatous scleritis. Clinical and histological features. Am J Ophthalmol 1967; 64: 1125-34.

7 Young RD, Watson PG. Microscopical studies of necrotising scleritis. II. Collagen degradation in the scleral stroma. $\mathrm{Br} \mathrm{J}$ Ophthalmol 1984; 68: 781-789.

8 Manschott WA. The eye in collagen diseases. Adv Ophthalmol 1961; 11: 1 .

9 Ashton N, Hobbs HE. Effect of cortisone on rheumatoid nodules of the sclera (scleromalacia perforans) BrJ Ophthalmol 1952; 36: 373-84.

10 Padawer J. Mast cell structure: Implications for normal physiology and degranulation. In: Pepys J, Edwards AM, eds. The mast cell its role in health and disease. London: Pitman Medical, 1979: 1-8.

11 Henriques AS, Bloch KJ, Kenyon KR, Baird RS, Hanninen LA, Allansmith $M$. Ultrastructure of mast cells in rat ocular tissue undergoing anaphylaxis. Arch Ophthalmol 1983; 101: 1439-46.

12 Fell HB, Jubb RW. The effect of synovial tissue on the breakdown of articular cartilage in organ culture. Athritis Rheum 1977; 20: 1359-71.

13 Saklatvala J, Sarsfield SJ. Lymphocytes induce resorption of cartilage by producing catabolin. Biochem J 1982; 202: 275-8.

14 Taylor D, Yoffe J, Woolley D. Histamine $\mathrm{H}_{2}$ receptors on foetalbovine articular chondrocytes. Biochem J 1983; 212: 517-20. 